\title{
X-RAY LUMINOUS NON-SEYFERT GALAXIES
}

\author{
M.J. WARD and D.H.HUGHES \\ Astrophysics, University of Oxford, Keble Road, Oxford OX1 3RH, U.K. \\ J.S. DUNLOP \\ Dept. of Chemical and Physical Sciences, Liverpool JM University, U.K. \\ and \\ P.N. APPLETON \\ Dept. of Physics and Astronomy, Iowa State University, U.S.A.
}

\begin{abstract}
There are now well established classes of X-ray emitting galaxies, which exhibit characteristic ranges of luminosity, for example Green et al. (1992). The ROSAT all sky survey, has detected a relatively small number of galaxies whose overall properties do not appear consistent with any of these classes. We discuss whether these X-ray luminous galaxies (XLGs) are the extreme end of the luminosity range of starburst galaxies, or whether they represent a new X-ray class of AGN.
\end{abstract}

Recently the ROSAT all sky survey has been cross-correlated with about 15,000 galaxies from the IRAS Point Source Catalog, resulting in detections of 244 galaxies (Boller et al., 1992). In addition to the recognized classes of AGN, starbursts and normal galaxies, this survey revealed a small number of X-ray luminous nonSeyferts exhibiting soft X-ray luminosities between $10^{41.5-43} \mathrm{ergs} \mathrm{s}^{-1}$. The top end of this range is comparable to Seyfert 1s, and the lower end is similar to Seyfert 2s, and the most luminous starburst galaxies detected before ROSAT (Green et al., 1992).

First we must offer a word of caution. Published classifications of AGN types based on optical spectroscopy, can be in error for a variety of reasons. From our high quality optical spectroscopy we note three cases of misclassification in the original list of XLGs, Boller et al. (1992). IRAS0628+63, IRAS1537-18 and IRAS132238, are all in fact Seyfert 1s. In view of this, their X-ray luminosities are not exceptional. However there still remain a number of XLGs whose optical spectra do not resemble Seyferts, see Fig. 1. In Table I, we list the properties of a subset of these non-Seyferts, together with IRAS1537-18 for comparison. Their X-ray luminosities are more than an order of magnitude higher than found for normal galaxies, Fabbiano et al. (1992).

Fruscione and Griffiths (1991) identified a number of Einstein selected galaxies, as X-ray luminous starbursts. Their conclusion, based on very few examples, was that starburst galaxies could account for at least $15 \%$ of the extragalactic background at $2 \mathrm{keV}$. Some of the XLGs discovered by ROSAT are even more luminous than those identified by Fruscione and Griffiths. In order to quantify their activity we plot a set of emission line ratio diagrams in Fig. 2.

The X-ray emission from normal (non-AGN) galaxies originates predominantly from the bulge component in early type spirals, and from the disk component in

311

T. J.-L. Courvoisier and A. Blecha: Multi-Wavelength Continuum Emission of AGN, 311-314.

(C) 1994 IAU. Printed in the Netherlands. 


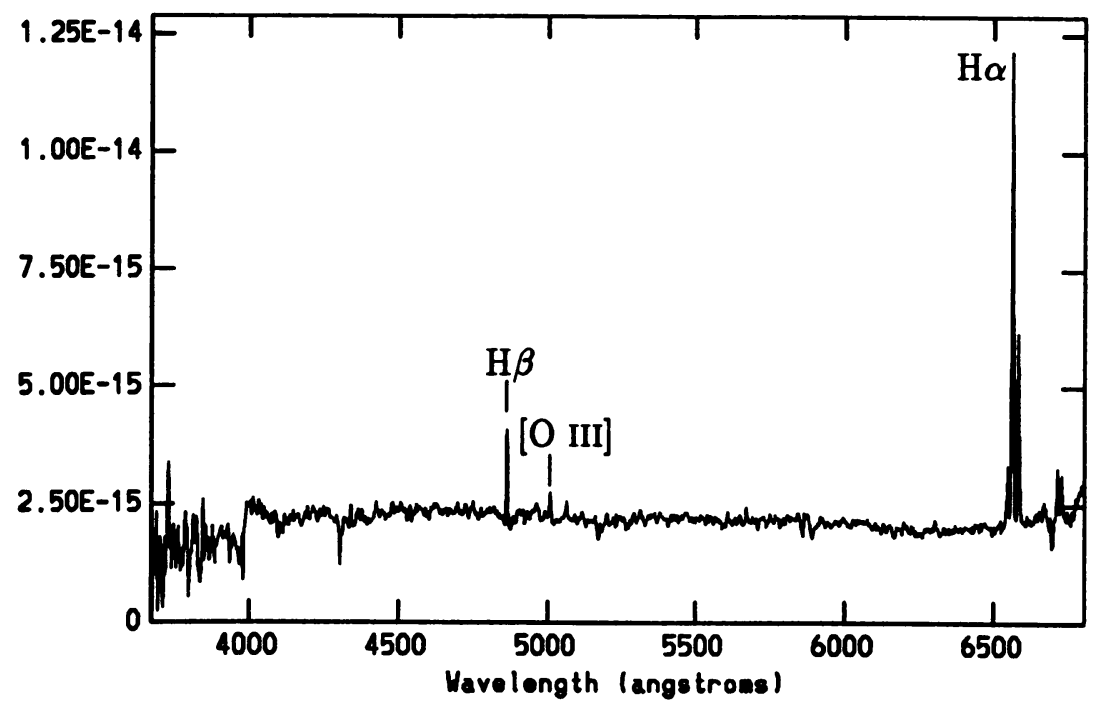

Fig. 1. Optical spectrum of IRAS $16155+6831$, showing it is a typical starburst/HII region galaxy. The flux scale is ergs $\mathrm{cm}^{-2} \mathrm{~s}^{-1}$. Compare with NGC 5248 in Kennicutt, (1992).

late-type spiral galaxies. However, a scaling of these stellar components shows that they fail to account for the X-ray emission from XLGs. Therefore in an attempt to explain their high X-ray luminosities we examine two propositions: either they are some variation on the theme of obscured AGN, following the Seyfert 1/Seyfert 2 unification scheme, or they are examples of powerful starburst activity at an extreme level previously unrecognized.

TABLE I

\begin{tabular}{ccccc}
\hline source & $\begin{array}{c}\log \mathrm{L}(\mathrm{X}-\mathrm{ray}) \\
\left(\begin{array}{c}0.1-2.4 \mathrm{keV}) \\
\mathrm{ergs} \mathrm{s}^{-1}\end{array}\right.\end{array}$ & $\begin{array}{c}\log \mathrm{L}(\mathrm{FIR}) \\
\left(\begin{array}{c}120 \mu \mathrm{m}) \\
\mathrm{ergs} \mathrm{s}^{-1}\end{array}\right.\end{array}$ & $\begin{array}{c}\log \mathrm{L}(\mathrm{H} \alpha) \\
\lambda 6563 \AA \\
\text { ergs s}^{-1}\end{array}$ & $\begin{array}{c}\log \mathrm{L}([\mathrm{OIII}]) \\
\lambda 5007 \AA \\
\text { ergs s }^{-1}\end{array}$ \\
\hline IRAS11395+1033 & 42.28 & 44.54 & 40.9 & 40.5 \\
IRAS12393+3520 & 42.80 & 44.22 & 39.8 & 40.1 \\
IRAS15374-1817 & 42.40 & 44.00 & 40.5 & 40.0 \\
IRAS16155+6831 & 42.39 & 44.36 & 41.3 & 40.1 \\
\hline
\end{tabular}

Considering the first possibility, it is unlikely that the low energy X-ray (Einstein and ROSAT) emission is due to transmitted radiation from an obscured AGN. This is because a foreground extinction of $A_{V} \gtrsim 5 \mathrm{mag}$ would be required to obscure the broad wings on the Balmer lines (e.g. Blanco, Ward \& Wright 1990). The asso- 


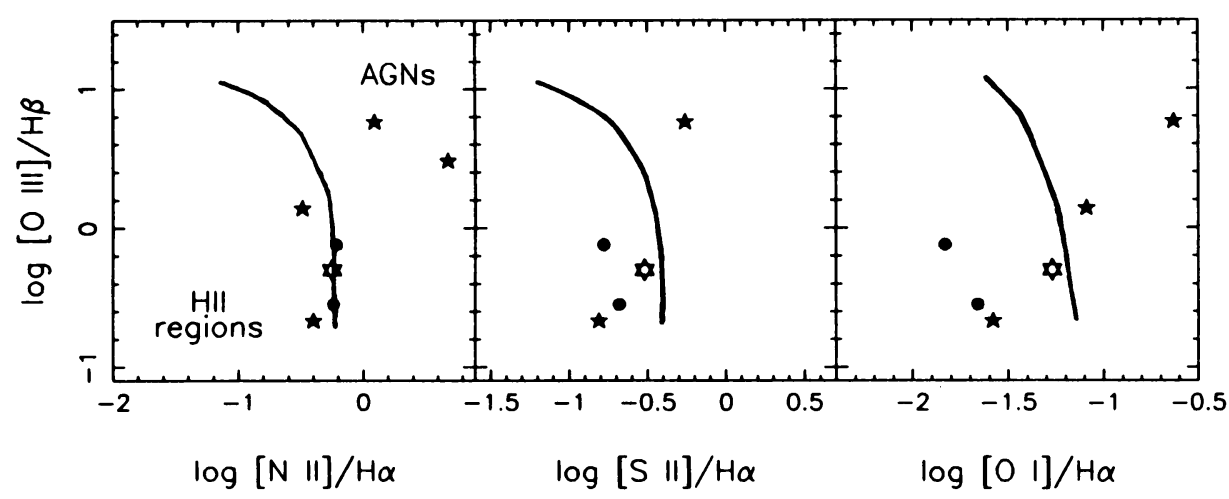

Fig. 2. Diagnostic emission line ratio diagrams. The solid line divides typical AGN from typical starburst/HII regions. Symbols are; filled stars for galaxies in table 1, filled circles for NGC 1614 and NGC 3256, previously known well-studied starburst galaxies with luminosities between $10^{41.3-42}$ ergs s $^{-1}$. The open star is for $1 \mathrm{E2251-17}$, one of the most X-ray luminous starburst galaxies detected by Einstein, with $10^{43} \mathrm{ergs} \mathrm{s}^{-1}$. Note: not all galaxies have measured ratios in all three plots.

ciated gas column of $\simeq 10^{22} \mathrm{~cm}^{-2}$ would easily extinguish the soft $X$-ray emission. A hidden AGN could produce detectable X-ray emission through electron-scattering as in the generally accepted model for Seyfert 2s. However, because of their high $\mathrm{X}$-ray luminosities (10-100 times that of a typical Seyfert 2), the scattering efficiency would have to be correspondingly high, but still not high enough for the scattered Broad Line Region to be detected in the optical spectrum. This might be possible with a scattering efficiency of around $10 \%$. Polarization measurements would settle this question.

Turning now to the super-starburst hypothesis, Fabbiano, Trinchieri \& MacDonald (1984) showed that in irregular blue galaxies undergoing bursts of star formation the ratio of $f_{X} / f_{B}$ was greater than in normal galaxies, with some notably extreme cases (Fabian \& Ward, 1993).

Hence energetic, widespread starburst activity is one way in which the X-ray emission can be significantly enhanced relative to the optical. Exactly how this is achieved is still a matter of debate. Evolved low-mass X-ray binaries would contribute starlight and X-rays proportionally, so appear to be ruled out by the high $L_{X} / L_{B}$ ratios. This is demonstrated graphically in Fig. 3, which shows the similarity of a XLG to a typical HII region galaxy, in all but its X-ray properties. Ohashi et al. (1990) favor thermal emission from gas at $\mathrm{kT} \simeq 1-3 \mathrm{keV}$ to fit their GINGA spectra of NGC253 and M83. The diffuse component in those galaxies could be fueled by supernovae and their remnants, but for the XLGs the numbers would need to be exceptional.

A plausible alternative would be a strong contribution from luminous, shortlived High Mass X-ray Binaries which display similar spectral characteristics. Such 


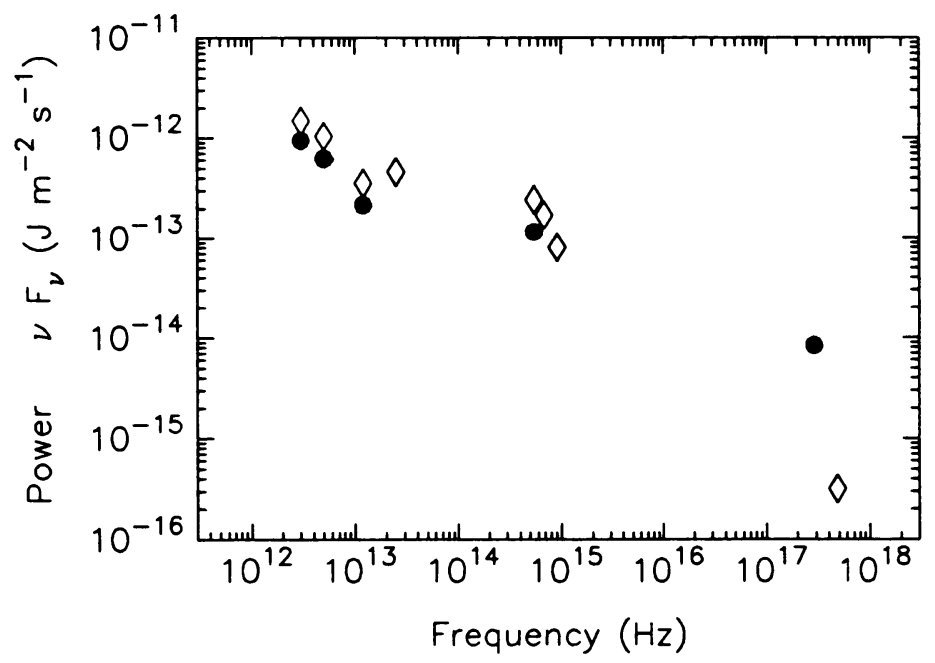

Fig. 3. The energy distribution, from far IR to X-rays, of IRAS $16155+6831$ (filled circles), compared with a typical starburst galaxy NGC 5248 (open diamonds). The fluxes for IRAS $16155+6831$ have been scaled up by a factor 10 , to facilitate comparison.

a short-lived population would enhance the X-ray/photospheric luminosity ratios of XLGs relative to quiescent galaxies (David et al., 1992). A difficulty is the requirement that a high percentage of all $O$-stars form in these systems.

In summary, ROSAT has detected a small but significant number of nonSeyferts, with high X-ray luminosities. A few are misclassified AGN, others may be LINERs, but some do appear to be starburst/HII region galaxies. Unless they evolve strongly, they probably do not contribute significantly to the X-ray background. Nevertheless, their X-ray luminosities do present problems for starburst models. X-ray spectra to define absorption cut-offs and temperatures, radio observations to quantify the SNR component, and measurement of the spatial extent of the starburst, are the next logical steps in this study.

\section{References}

Blanco, P., Ward, M.J.,\& Wright, G.S., 1990, Mon. Not. R. astr. Soc., 242, 4p

Boller, Th., Meurs, E.J.A., Brinkmann, W. et al., 1992, Astron. Astrophys. Suppl., 261,57

David, L.P., Jones, C. \& Forman, M.J., 1992, Astrophys. J., 388, 82

Fabian, A.C. \& Ward, M.J., 1993, Mon. Not. R. astr. Soc., 263, L51

Fabbiano, G., Trinchieri, G. \& MacDonald, A., 1984, Astrophys. J., 284, 65

Fabbiano, G., Kim, D.-W. \& Trinchieri, G., 1990, Astrophys. J. Suppl., 80, 531

Fruscione, A. \& Griffiths, R., 1991, Astrophys. J., 380, L13

Green, P.J., Anderson, S.F. \& Ward, M.J., 1992, Mon. Not. R. astr. Soc., 254, 30

Kennicutt, R.C., 1992, Astrophys. J. Suppl., 79, 255

Ohashi, T. et al., 1990, Astrophys. J., 365, 180. 